\title{
Curcumin Induces Human Glioma Cell Apoptosis by Promoting Reactive Oxygen Species Production
}

\author{
YU ZHOU*AND LI LIU1 \\ Department of Neurosurgery, ${ }^{1}$ Department of Health Examination, Tianjin Huanhu Hospital, Tianjin 300350, China
}

\author{
Zhou et al.: Curcumin Induces Human Glioma Cell Apoptosis
}

\begin{abstract}
Malignant glioma by surgery is difficult to completely remove, the recurrence rate is high, the survival period is short and the prognosis is poor, which poses a great threat to human health. This article mainly studies the related process that curcumin induces human glioma cell apoptosis by promoting reactive oxygen species production. In the experiment, glioma cells were processed and Uppsala 87 malignant glioma cells of human were cultured in vitro. 3-(4, 5-dimethylthiazolyl-2)-2, 5-diphenyltetrazolium bromide assay was used to detect the effect of curcumin on the proliferation of Uppsala 87 malignant glioma cells. The cells in each reagent group and control group treated with curcumin were treated with 3-(4, 5-dimethylthiazolyl-2)-2, 5-diphenyltetrazolium bromide colorimetric treatment at $24 \mathrm{~h}$. Use flow cytometry to detect cell apoptosis. Cellular immunofluorescence was used to detect the effect of oxidase inhibitors on nuclear factor activation. Experimental data showed that the apoptotic rate of 0, 2.5, $5 \mathrm{mg} / \mathrm{l}$, diallyl disulphide treatment groups were $12.3 \%, 19.75 \%$ and $26.44 \%$, respectively, indicating that diallyl disulphide caused an increase in the apoptotic rate of human promyelocytic leukemia cell line cells and the difference was statistically significant $(p<0.05)$. The results show that curcumin can inhibit the proliferation of Uppsala 87 malignant glioma cells cultured in vitro, induce the differential expression of apoptosis-related genes and thus have a significant effect of promoting cell apoptosiss.
\end{abstract}

Key words: Curcumin crystals, reactive oxygen species, human glioma cells, flow cytometry

Gliomas are the most common malignant tumors in the brain. Among them, glioblastoma multiforme (GBM) is the most common in adults, accounting for 29.6 $\%$ of intracranial tumors. The degree of malignancy is extremely high and the prognosis of the patient is extremely poor. The current treatment methods include: surgical resection, radiotherapy, chemotherapy, cellular immunotherapy and other comprehensive treatment methods. As far as the current treatment plan is concerned, the first choice is still surgical resection, which can not only remove the lesion to the maximum extent, but also clarify the pathology and guide postoperative radiotherapy and chemotherapy. However, the clinical prognosis of patients is still not optimistic with the maximal surgical resection and postoperative radiotherapy and chemotherapy. More critically, the standard alkylating agent chemotherapeutic drug temozolomide can induce cell mutations while inhibiting the proliferation of tumor cells; long-term large-scale use increases the probability of tumor recurrence and leads to a significant increase in the gene mutation rate in recurrent tumor tissues.

*Address for correspondence E-mail: pjbsy8566@163.com

July-August 2021
Curcumin can exert anti-tumor effects through a variety of ways, such as inducing mutations and oncogene expression to affect the occurrence and development of tumors and has no toxic side effects on normal cells. It has also been found that curcumin can also enhance sensitization of radiotherapy and chemotherapy and apoptosis molecular targets to the effect of treatment; detect changes in the intensity of fluorescent probes by flow cytometry, measure intracellular production and detect cell apoptosis and explore the effects of curcumin on the proliferation, survival, apoptosis and intracellular production of glioma cells. It is of exploratory significance to analyze the recurrence of glioma.

Curcumin has a significant promoting effect on cell apoptosis. Surulescu believes that glioma is a wide

This is an open access article distributed under the terms of the Creative Commons Attribution-NonCommercial-ShareAlike 3.0 License, which allows others to remix, tweak, and build upon the work non-commercially, as long as the author is credited and the new creations are licensed under the identical terms

Accepted 15 July 2021

Revised 18 November 2020

Received 10 March 2020

Indian J Pharm Sci 2021;83(4):714-722 
range of brain and spinal cord tumors caused by glial cells, which are the main brain cells that can develop into tumors. He proposed a multi-scale model of glioma growth, including the interaction of cells and basic tissue networks and proliferation. He explained the two subgroups of cells to adapt to proliferation based on the dichotomy of cell growth or division. Although his research is theoretically correct, it lacks pertinence ${ }^{[1]}$.

$\mathrm{Yu}$ developed a multifunctional fusion protein for targeting therapeutic elements and delivering them to glioma cells. He found that the multifunctional fusion protein is expressed in Escherichia coli. He used nickel-nitrilotriacetic acid (Ni-NTA) resin affinity chromatography for purification. He used human glioma cells and primary astrocytes to analyze their functions. He observed through a confocal microscope that the targeted protein was localized to glioma cells. He used the cell counting kit 8 and the colony formation assay to evaluate the effects of cell viability and proliferation. He uses the transwell assay to assess the migration and invasion of glioma cells and analyzes apoptosis by flow cytometry. In addition, he used western blotting to measure changes in protein expression related to cell cycle and apoptosis. His research is relatively comprehensive, but lacks the necessary experimental data $^{[2]}$.

Zhou believes that microRNA (miRs) is a type of non-coding RNA. He found that miR-181b plays an inhibitory role in glioma, but the regulatory mechanism of miR-181b in the malignant phenotype of glioma cells is still unclear. He found that compared with normal brain tissue, miR-181b in glioma tissue was significantly down-regulated and the decrease in miR$181 \mathrm{~b}$ level was significantly related to the high pathology and poor prognosis of patients with glioma. Although his research has certain reference significance, but the factors considered are not comprehensive $\mathrm{e}^{[3]}$.

In this paper, by studying the process of human glioma cell apoptosis, the molecular mechanism of curcumin on cell apoptosis is deeply analyzed. By establishing a biochemical reaction system and establishing an in vitro cell oxidative damage model, the role of curcumin in promoting the oxidation mechanism and inducing human Uppsala 87 (U87) malignant glioma cell apoptosis was discussed in depth. After reducing cell oxidative phosphorylation in many tumor tissues, the growth and invasion capabilities of tumor cells are significantly enhanced. It can be seen that the changes in mitochondrial structure and function have profound effects on the proliferation, energy metabolism, differentiation and apoptosis of tumor cells.

\section{CURCUMIN AND GLIOMA CELL APOPTOSIS}

\section{Curcumin and reactive oxygen species (ROS):}

Curcumin is a polyphenol compound, mainly extracted from turmeric, a plant of the turmeric family, which can make vanilla yellow. Turmeric is mainly cultivated in tropical and subtropical regions. The curcumin in turmeric is extracted from turmeric by solvent extraction methods such as paraffin wax and ultrasonic and refined by colorimetry. Curcumin is a natural substance extracted from plants, with a wide range of efficacy and it may develop into a natural antibiotic in the future ${ }^{[4]}$. The pharmacological effects of curcumin are as follows.

Anti-inflammatory effect: For example, it has obvious anti-inflammatory effects on joint rheumatism, pancreatitis, allergic meningitis and other inflammatory diseases.

Antioxidant effect: Curcumin reduces the content of lipoproteins in the blood, thereby reducing blood lipids and anti-atherosclerosis. The lowering of lipids in the body will also change the metabolism of fatty acids, promote the metabolism of low-density lipoprotein (LDL) and lipoproteins in the liver and adrenal glands, increase the excretion of LDL in the gallbladder and hinder the intake of LDL in the spleen.

Role of capturing anaerobic free radicals: Curcumin can improve the tolerance of myocardium to hypoxia, has a certain protective effect on myocardial ischemic injury, improves the activity of superoxide catalase and can effectively remove superoxide compounds that have a protective effect on ischemia reperfusion disorders.

Protective effect on the liver: In in vivo and in vitro, it has a protective effect against liver disorders caused by various poisons such as carbon tetrachloride, aflatoxin B1, iron and cyclophosphamide and greatly reduces phospholipids in liver tissue. Therefore, curcumin has the effect of preventing fatty liver.

Anti-fibrosis effect: Inhibit the release of tumour necrosis factor alpha (TNF- $\alpha$ ) and the production of anions and nitric oxide (NO). This means that curcumin may have two very effective chemicals in vivo and in vitro.

Anti-cancer effect: It can make a variety of tumor cells 
gather in the synthesis phase (S phase) and cannot enter the next cell proliferation and division cycle, which has a significant inhibitory effect on $\mathrm{it}^{[5]}$.

Curcumin, demethoxycurcumin (DMC) and bisdemethoxycurcumin (BDMC) are the polyphenols in turmeric rhizome extracts with antioxidant activity. Curcumin can inhibit carcinogenic ROS such as nitrite free radicals. In the treatment of patients with advanced colon cancer, curcumin can effectively reduce the forkhead transcription factor 3 in regulatory $\mathrm{T}$ cells (Tregs) and increase the working frequency of type $1 \mathrm{~T}$ helper (Th1) cells.

As the main part of oxidative phosphorylation in eukaryotes, mitochondria have (adenosine triphosphate (ATP)) ATPase complex and four respiratory chain enzyme complexes attached to their inner membrane. The respiratory chain is the most important part of the cell to produce ROS. $95 \%$ of ROS in normal cells originate from this. In addition to mitochondria and nicotinamide adenine dinucleotide phosphate (NADPH) oxidase, the intracellular ROS can also generate ROS through some chemical reactions in the cytoplasmic matrix. Under physiological conditions, in order to balance the redox state in the cell, the body will always rely on a complete and efficient redox regulation mechanism to tightly regulate the balance state ${ }^{[6]}$.

Under normal blood glucose level, retinal pigment epithelium (RPE) cells can secrete a variety of small amount of cytokines, such as interleukin 1 beta (IL-1 ), TNF- $\alpha$, intercellular adhesion molecule 1 (ICAM-1), vascular endothelial growth factor(VEGF), transforming growth factor beta 1 (TGF- $\beta 1$ ) etc., to maintain the normal structure and function of the retina; while in the high glucose environment, RPE cells under the stimulation of excessive ROS, the content of the above cytokines can be abnormal, thus affecting the normal function of the retina. RPE cells induced by high glucose can produce a large amount of ROS, which can activate nuclear factor kappa B (NF- $\kappa \mathrm{B})$ in cells, amplify oxidative stress and inflammatory reaction in RPE cells and jointly promote oxidative damage and fibrosis of RPE cells ${ }^{[7]}$.

\section{Glioma:}

Glioma is very special in terms of cell origin, pathological structure, biological characteristics and clinical symptoms. It rarely metastasizes to organs other than the skull and hardly spreads or grows in the brain. Glioma grows like a non-capsular infiltrate. At present, surgical treatment is still the first choice for the treatment of glioma, but the surgical treatment is very poor, the trauma is serious and it is often difficult to achieve true and complete resection due to unclear boundaries. The recurrence is fast and the degree of malignancy often increases after recurrence. Glioma cells have a larger colony in the dormant phase of the cell proliferation cycle and the tumor cell membrane has more lipid components and conventional nucleic acidtargeted cytotoxic chemotherapy and radiotherapy are highly specific to destructive glioma cells. In addition, the central nervous system may have systemic adverse reactions. At the same time, the tumor cells in the tumor have multiple heterogeneity and weak immunogenicity, which makes it impossible for various immunotherapy and molecular surgery techniques to conduct more effective clinical application research ${ }^{[8]}$.

The occurrence and development of malignant glioma are the result of multiple factors, these factors include; loss of function of tumor suppressor genes phosphoprotein (p53) and phosphatase and tensin homolog (PTEN); loss of function of cell division cycle inhibitor genes, such as p16 and p19; genes encoding growth factor receptors proliferation and mutation; activation of related genes and proteins leads to the production of growth regulatory proteins, such as protein kinase $\mathrm{C}$. These proteins play an important role in tumor cell generation, proliferation, metastasis and tolerance to radiotherapy and chemotherapy. However, the mechanism of how these signal proteins maintain the survival and proliferation of glioma cells is not very clear and may be related to the anti-apoptotic mechanism of glioma cells ${ }^{[9]}$. Phosphoinositide 3-kinase (PI3K)/ protein kinase $\mathrm{B}$ (Akt) pathway, transcription factor $\mathrm{NF}-\kappa \mathrm{B}$, mammalian target of rapamycin (mTOR) can enhance the anti-apoptotic effect of glioma cells. At the same time, studies have also found that ROS act on targets such as PI3K/Akt and transcription factor NF$\kappa \mathrm{B}$, which play an important regulatory role in signal transduction pathways that maintain cell survival and proliferation. At present, the main treatment method for glioma is to remove the tumor as widely as possible while protecting the function of the nervous system to the maximum extent and then perform postoperative radiotherapy and chemotherapy. During surgery, tumors will affect normal brain tissue and make it infiltrate, so complete removal is often difficult. After surgery, due to the high proliferation of tumor cells, the slowing down of apoptosis and the growth of new blood vessels, the glue mass tumors often recur within a short period of time after surgery. Therefore, the current treatment of glioma is facing huge difficulties ${ }^{[10]}$. 


\section{Apoptosis:}

There are two different forms of cell death in animals. One is mainly due to cell necrosis caused by some external factors such as ischemia, physical and chemical damage or rapid cell death caused by biological infiltration and the other main form is cell death. Apoptosis is called programmed cell death. Cells undergo a process of self-regulated death by body genes and are eroded and removed by other cells. Apoptosis is very important in the process of life. It is a necessary condition for the survival of multicellular organisms and runs through the entire life process of organisms. Apoptosis is an autonomous procedural process regulated by genes, usually without inflammation, mainly to maintain the homeostasis of the cellular environment. Autophagy is a process in which cells engulf their own components and fuse with lysosomes to form autophagic vesicles, digest and degrade phagocytic components. Apoptosis and autophagy are a mutual regulation relationship that promotes and antagonizes each other. Autophagy has a dual effect on tumor cells. On the one hand, autophagy can cause autophagic cell death, inhibit the growth of tumor cells and have a defensive effect on certain tumors. On the other hand, tumor cells can reduce hypoxia and reduce hypoxia through autophagy. Nutrient deficiency, mitochondrial damage, oxygen free radicals and other stress damage cells, forms a hibernation-like state to protect tumor cell survival and promote tumor growth. These may be the drug resistance mechanism of tumors. The effect of autophagy on tumor cells has not yet been determined and whether to induce autophagy or inhibit autophagy to kill tumor cells depends on the genotype of tumor cells and its treatment methods ${ }^{[11,12]}$.

\section{MATERIALS AND METHODS}

\section{Human glioma cell apoptosis experiment:}

Experimental materials and instruments: The main materials used in the experiment are human glioma U87 cell line and curcumin powder. The main instruments are ultra-clean workbench, automatic carbon dioxide $\left(\mathrm{CO}_{2}\right)$ constant temperature incubator, inverted microscope, high-speed low temperature centrifuge, polymerase chain reaction (PCR) amplification instrument, etc.

Reagent preparation: Roswell Park Memorial Institute medium (RPMI 1640) complete culture medium: $9.8 \mathrm{~g}$ RPMI 1640 medium powder and $2.0 \mathrm{~g}$ sodium bicarbonate powder were dissolved in $1000 \mathrm{ml}$ double distilled water and the packaging bag was washed with double distilled water for 2-3 times. The washing solution was added to the culture medium, stirred by magnetic stirrer for 30-60 min until the powder was completely dissolved. Adjust the $\mathrm{pH}$ value to 7.2-7.4 with $1 \mathrm{mmol} / 1$ salt acid or sodium hydroxide. Take Chua's filter and filter and remove bacteria with 0.22 $\mu \mathrm{m}$ filter membrane in the ultra-clean platform. After sub packaging, take a small amount of culture medium to $\mathrm{CO}_{2}$ incubator for bacteria detection and then store it in refrigerator at $-20^{\circ}$ for future use.

$10 \%$ fetal bovine serum (FBS) is prepared. $0.22 \mu \mathrm{m}$ filter membrane was used to remove bacteria. After sub-packaging, take a small amount of culture solution to the $\mathrm{CO}_{2}$ incubator for bacteria detection and store it in the refrigerator at $-20^{\circ}$ for later use. Immediately before use, add RPMI 1640 culture medium at a final concentration of $10 \% \mathrm{FBS}$.

$1.5 \%$ agarose gel was prepared. Weigh $1 \mathrm{~g}$ of agarose gel with an electronic balance and place it in a glass beaker, then add $100 \mathrm{ml} \quad 0.5 \times$ Tris-borate-ethylenediamine tetraacetic acid (TBE) to it, shake the glass beaker to make it initially mixed and then put it in a microwave oven for heating. Make the agarose powder completely dissolve, then add $3 \sim 5 \mu 1$ of $10 \mathrm{mg} / \mathrm{ml}$ ethidium bromide (EB) solution to the solution and mix well, pour the solution into the glue board and spread the glue.

$1 \%$ Diethyl pyrocarbonate (DEPC) treated water was prepared. Use a micropipette to draw $1000 \mu 1$ of DEPC and add it to $1000 \mathrm{ml}$ of deionized water, stir well with a glass rod and then high pressure treatment at $121^{\circ}$. After cooling, store it at $4^{\circ}$ for later use.

Cell processing: Cell culture-Take out the cryopreserved glioma U87 cells from the liquid nitrogen tank, preheat it in a water bath for 3 to $5 \mathrm{~min}$, wait until the cryopreservation solution of the cells in the cryopreservation tube is completely dissolved, then suck the cryopreservation solution in the ultra-clean bench. Put it in a $15 \mathrm{ml}$ centrifuge tube, centrifuge for $5 \mathrm{~min}$, absorb the supernatant, put $1 \mathrm{ml}$ of pre-warmed complete medium containing $10 \%$ FBS into the centrifuge tube, mix the cells and transfer to the culture plate. After adding $5 \mathrm{ml}$ of the culture solution to the culture solution, incubate in a $37^{\circ}, 5 \% \mathrm{CO}_{2}$ incubator. Change the medium in time and keep the humidity in the incubator. Pancreatin digestion time cannot be too long. Cell recovery-After removing the frozen cells from liquid nitrogen, quickly put them in a $37^{\circ}$ water bath. After the frozen storage liquid has dissolved more than $50 \%$, it is transferred to the Iscove's modified 
Dulbecco's medium (IMDM) culture site preheated at $37^{\circ}$. Gently paste the cells with a pipette, centrifuge at $800 \mathrm{rpm}$ for $5 \mathrm{~min}$ and wash once with phosphatebuffered saline (PBS). Then add IMDM medium, transfer it to the culture flask evenly with a pipette and put it in a constant temperature incubator at $37^{\circ}$.

\section{3-(4,5-dimethylthiazolyl-2)-2,5-diphenyltetrazolium bromide (MTT) detection:}

The culture flask of U87 cells was placed under the inverted microscope to observe the cell attachment and confirm that the U87 cells are in the logarithmic phase. The cells were counted with the detectivity quantum counter $96 \mu \mathrm{m}$ and the cells were counted with microscope. When the cells were dispersed and even, 96 well plates were placed in cell incubator $\left(37^{\circ}, 5 \% \mathrm{CO}_{2}\right)$ and incubated overnight. After curcumin treatment, the cells of each reagent group and control group were treated with MTT colorimetry at $24 \mathrm{~h}$. MTT $20 \mu \mathrm{l}(5$ $\mathrm{mg} / \mathrm{ml}$ ) was added into each experimental hole of 96 well plate and cultured in $5 \% \mathrm{CO}_{2}$ incubator at $37^{\circ}$ for $4 \mathrm{~h}$. After centrifugation, $200 \mu \mathrm{l}$ dimethylsulfoxide (DMSO) was added and fully vibrated for $15 \mathrm{~min}$. The optical density (OD) value, average OD value and standard deviation of each group were detected and recorded by automatic enzyme marker (detection wavelength: $570 \mathrm{~nm}$ ).

Double staining to detect apoptosis: U87 cells in each group were cultured continuously for $48 \mathrm{~h}$ and then digested with $0.25 \%$ trypsin, collected in a centrifuge tube, centrifuged to discard the supernatant. Add a single cell suspension containing serum-free DMEM cell medium and then count the number of cells. After trypsin digestion, the culture medium stops digestion and collects cells. Wash once with PBS (containing $0.3 \%$ bovine serum albumin (BSA)), collect cells by centrifugation at $2000 \mathrm{r} / \mathrm{min}$ for $5 \mathrm{~min}$, wash once with physiological PBS and collect cells by centrifugation at $1800 \mathrm{r} / \mathrm{min}$ for $4 \mathrm{~min}$. Add $200 \mu \mathrm{l}$ of physiological PBS containing $4 \mu \mathrm{g} / \mathrm{ml}$ acridine orange/ethidium bromide (AO/EB) staining solution to make cell suspension. Observe the cell morphology and take pictures under the IX71 fluorescence microscope.

\section{Statistical analysis:} data and use statistical package for the social sciences (SPSS) 20.0 statistical software for analysis. The measurement data is expressed as mean \pm standard deviation $(\mathrm{x} \pm \mathrm{s})$ and independent sample $\mathrm{t}$ is used in
Use Excel spreadsheet software to input experimental

the analysis of variance to test the mean between two samples to make comparisons between groups. If the difference between the groups is statistically significant, two comparative Student-Newman-Keuls pairwise studentized range (q) (SNK-q) tests are performed between the groups, $\mathrm{p}<0.05$ was considered statistically significant.

\section{RESULTS AND DISCUSSION}

Curcumin induces apoptosis of human glioma cells and the effect of curcumin on human glioma cells is shown here. MTT method was used to detect the effect of curcumin on glioma U87 cells. After treatment with curcumin, the cells were cultured in the incubator for $48 \mathrm{~h}$. The absorbance (OD value) of 96 well plate at 490 $\mathrm{nm}$ was determined by enzyme-linked immunosorbent assay (ELISA). The results are shown in Table 1 and fig. 1. Excessive production of ROS can destroy redox homeostasis, which may directly induce oxidative damage of lipids, proteins and nucleic acids, thereby killing cancer cells by interfering with metabolism and signal transduction of cancer cells. In addition, due to the barrier effect of biofilm, curcumin solution cannot reach the deep layer of biofilm or the arrival time is prolonged, so there is no enough curcumin to combine with bacteria, which may be one of the reasons for weak photodynamic killing effect. At the same time, after the formation of biofilm, the internal environment of biofilm is anoxic and acidic, lack of nutrients, many bacteria can carry out fixed stage dormancy, which leads to the weakening of the killing and inhibiting effect of methicillin-resistant Staphylococcus aureus (MRSA) in the biofilm. Curcumin group and photodynamic therapy (PDT) group had no effect on the survival rate and biofilm of MRSA, which indicated that photosensitizer and light of specific wavelength were indispensable factors in photodynamic killing effect. It also suggested that photosensitizer or corresponding wavelength irradiation did not have independent biological effect.

NADPH oxidase is the main source of ROS production in cells and it plays an important role in regulating cell proliferation, apoptosis and gene transcription. The research results show that the use of

TABLE 1: ABSORBANCE (OD VALUE) RESULTS

\begin{tabular}{lcccc}
\hline Concentration $(\mu \mathrm{g} / \mathrm{ml})$ & $\mathbf{2 . 5}$ & $\mathbf{5}$ & $\mathbf{1 0}$ & $\mathbf{2 0}$ \\
\hline 1 & 1.33 & 0.91 & 0.72 & 1.11 \\
2 & 1.04 & 0.67 & 0.54 & 0.87 \\
3 & 0.59 & 0.48 & 1.22 & 1.14 \\
4 & 0.46 & 0.57 & 0.81 & 0.77 \\
5 & 0.97 & 0.79 & 0.29 & 1.06 \\
\hline
\end{tabular}


diphenyleneiodonium (DPI) can effectively inhibit the activity of NADPH oxidase and significantly reduce the production of intracellular ROS, indicating that NADPH oxidase 4 (NOX4) may be the most important enzyme body for ROS production in glioma cells. Human malignant glioblastoma multiforme (U251) glioma cells were treated with different concentrations of NADPH oxidase inhibitor DPI $(5,15,25 \mu \mathrm{m})$ and antioxidant Tiron $(10 \mathrm{~mm})$. After $24 \mathrm{~h}$, the cell viability was detected by the alamarBlue method. The results showed that DPI and Tiron both significantly inhibit the growth of J251 glioma cells and the inhibitory effect of DPI on the proliferation of U251 glioma cells is dosedependent. After U251 cells were treated with DPI (25 $\mu \mathrm{M})$, the intracellular chromatin was concentrated, the nuclei divided and the cells showed the characteristics of programmed apoptosis. After treatment with different concentrations of DPI and Tiron, the apoptosis rate of U251 cells was significantly higher than that of the normal control group. These results indicate that NOX4 and its derived ROS participate in the regulation of signal transduction pathways such as the survival, growth and apoptosis of glioma cells. Enhancing the expression and activity of NOX4 in glioma cells and increasing the level of intracellular ROS play an extremely important role in promoting the growth of glioma cells and enhancing their anti-apoptotic ability and maintaining tumor cell survival (Table 1 and fig. 1).

Flow cytometry test results were shown here. Apoptotic rate and autophagy rate of human leukemia cell line (HL-60) cells were detected by flow cytometry combined with annexin V-propidium iodide (PI) double staining and flow cytometry combined with AO staining at the concentration of $0,2.5,5 \mathrm{mg} / \mathrm{l}$. The results are shown in fig. 2. The apoptotic rates of $0,2.5$, $5 \mathrm{mg} / 1$ diallyl disulphide (DADS) group were $12.3 \%$, $19.75 \%$ and $26.44 \%$, respectively, which indicated that DADS could increase the apoptosis rate of HL60 cells and the difference was statistically significant $(\mathrm{p}<0.05)$. The autophagy rate of $0,2.5,5 \mathrm{mg} / 1$ group was $88.87 \%, 98.98 \%$ and $93.13 \%$ respectively, which indicated that DADS induced the increase of autophagy rate of HL-60 cells and the peak was in $2.5 \mathrm{mg} / \mathrm{l}$ group, the difference was statistically significant $(\mathrm{p}<0.05)$. These results suggest that DADS induce autophagy to apoptosis in HL-60 cells in a concentration threshold dependent manner. Autophagy was increased in low concentration $(2.5 \mathrm{mg} / \mathrm{l})$ of DADS, while apoptosis was increased in high concentration $(5 \mathrm{mg} / \mathrm{l})$. Only when the concentration of DADS reaches a certain threshold, can HL-60 cells change from induced autophagy to apoptosis, from quantitative change to qualitative change. The expression of proliferating cell nuclear antigen (PCNA) protein in human glioma cells was significantly higher than that in normal glioma cells and the higher the malignant degree of glioma cells, the more significant the increase of PCNA protein expression. After curcumin treatment, the positive rate of PCNA protein decreased significantly compared with the control group, indicating that the synthesis of intracellular deoxyribonucleic acid (DNA) was significantly reduced and the proliferation of tumor cells was significantly inhibited, which was consistent with the results of MTT assay and plate colony formation test. These results indicate that curcumin can inhibit the proliferation of U251 cells in vitro (fig. 2).

Effect of curcumin and hydrogen peroxide $\left(\mathrm{H}_{2} \mathrm{O}_{2}\right)$ on ROS content in human Glioma U87 cells was described below. ROS detection results are shown in Table 2 and fig. 3 . The results showed that after curcumin treatment of $\mathrm{U} 87$ cells for $2 \mathrm{~h}$, the intracellular reactive oxygen level was significantly higher than that of the control group. After $10 \mu \mathrm{mol} / 1$ curcumin treated the cells, the reactive oxygen level reached nearly twice of the untreated cell group. After $20 \mu \mathrm{mol} / 1$ curcumin acts on cells and the level of ROS also increases significantly.

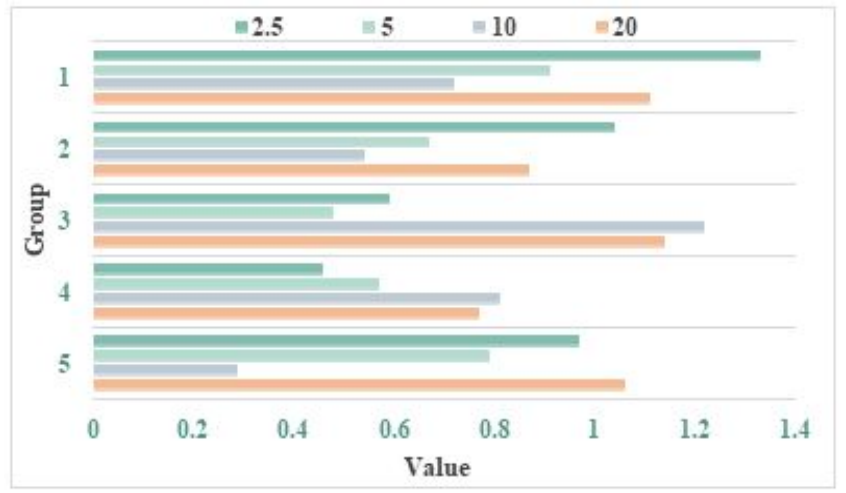

Fig. 1: Absorbance (OD value) results

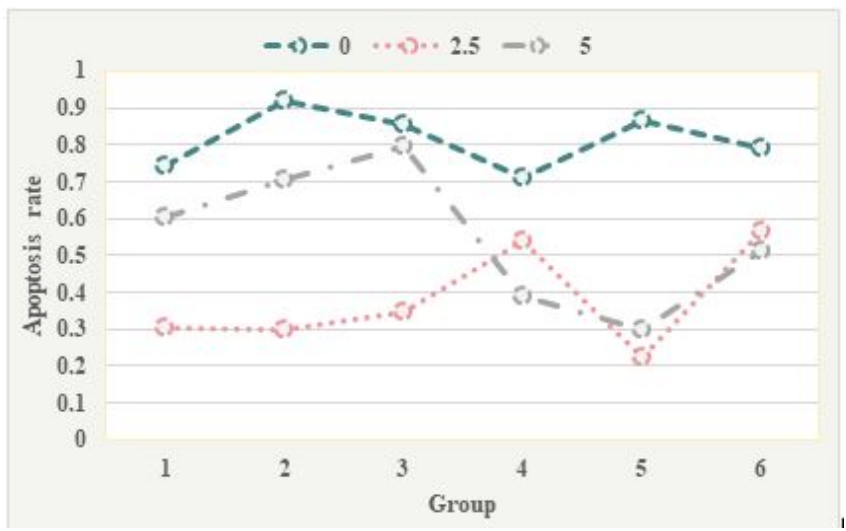

Fig. 2: Apoptosis rate and autophagy rate results when DADS concentration is $0,2.5,5 \mathrm{mg} / \mathrm{l}$ 
TABLE 2: ROS TEST RESULTS

\begin{tabular}{lcccccc}
\hline Concentration & $\mathbf{1}$ & $\mathbf{2}$ & $\mathbf{3}$ & $\mathbf{4}$ & $\mathbf{5}$ & $\mathbf{6}$ \\
\hline Creatine kinase (CK) & 1.22 & 1.03 & 1.11 & 0.49 & 0.55 & 0.47 \\
$10 \mathrm{C}$ & 1.19 & 0.77 & 1.30 & 1.01 & 0.26 & 0.55 \\
$20 \mathrm{C}$ & 1.04 & 0.59 & 0.61 & 0.83 & 1.22 & 1.06 \\
$100 \mathrm{H}$ & 0.69 & 0.71 & 0.44 & 1.03 & 0.57 & 0.89 \\
$10 \mathrm{C}+100 \mathrm{H}+20 \mathrm{C}+100 \mathrm{H}$ & 1.22 & 0.77 & 0.56 & 0.62 & 0.73 & 1.06 \\
\hline
\end{tabular}

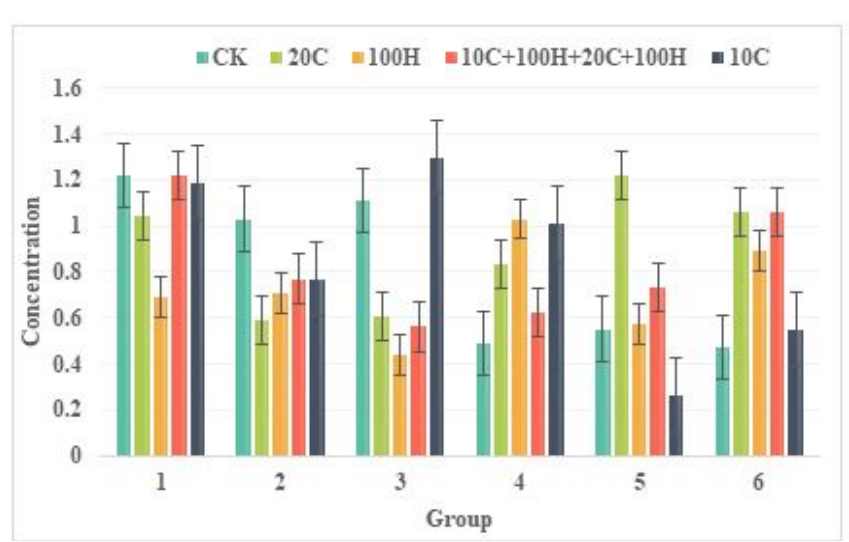

Fig. 3: ROS detection results

But $10 \mu \mathrm{mol} / 1,20 \mu \mathrm{mol} / 1$ curcumin combined with $\mathrm{H}_{2} \mathrm{O}_{2}$ treatment can also cause the level of ROS to increase after $2 \mathrm{~h}$ and the difference is not significant compared with the control group. After treating the cells for $6 \mathrm{~h}$, there was no significant difference between the treatment groups, indicating that the increase in ROS caused by curcumin had fallen back at $6 \mathrm{~h}$. Treatment of cells with $100 \mu \mathrm{mol} / 1 \mathrm{H}_{2} \mathrm{O}_{2}$ for $2 \mathrm{~h}$ or $6 \mathrm{~h}$ did not increase the level of intracellular ROS. This phenomenon suggests that curcumin may increase the level of ROS and cause oxidative damage to cells in a short time.

Glutathione (GSH) is an important metabolite in human cells and the main non-protein thiol-based compound in tissues. It removes superoxide ions and other free radicals in the body and has a lipid peroxidation effect. GSH can prevent the destruction of cell membranes by peroxides and free radicals, protect the integrity of cell membranes, protect intracellular proteins and DNA from free radicals and maintain normal cell metabolism. Increased GSH in tumor cells is a major cause of tumor cell resistance to anticancer drugs. ROS is active in nature and participates in the physiological and biochemical activities of cells or tissues very rapidly. It can directly participate in signal transduction between cells and affect a variety of cell pathways and the activation of inflammatory pathways is directly related to ROS. The experimental results show that curcumin mainly acts on PI3K/AKT and NF- $\mathrm{KB}$ pathways in tumor cells and there is an important connection between these two pathways. The activation of the PI3K/AKT pathway can activate the phosphorylation of inhibitor of NF- $\kappa \mathrm{B}(\mathrm{I} \kappa \mathrm{B})$ kinase (IKK) and IKK is an important regulator of NF- $\kappa B$. Its phosphorylation can promote the expression and phosphorylation of NF- $\kappa \mathrm{B}$, thereby increasing the downstream protein cyclooxygenase- 2 (COX-2) expression. Curcumin plays an important regulatory role in this process, that is, it inhibits the activation of the Akt pathway, thereby reducing the phosphorylation of the related proteins IKK and NF$\kappa \mathrm{B}$ in the inflammatory pathway and reducing the expression of COX-2. After the production of ROS, it can participate in various physiological activities in the body, including body defense, the synthesis of certain active substances, etc. At the same time, it plays an important role in the process of carcinogenesis, which can damage the DNA in the cell and promote the development of carcinogens. It is also a way for many carcinogens to exert their carcinogenic effects. However, under certain conditions, ROS can kill cancer cells and can induce apoptosis and necrosis of cancer cells (Table 2 and fig. 3).

The effect of different concentrations of curcumin on the enzyme activity of glioma cells is shown in fig. 4 . Compared with $0 \mu \mathrm{m}$ curcumin group $(2 \mu \mathrm{m}, 4 \mu \mathrm{m}, 6$ $\mu \mathrm{m})$, the relative expression of matrix metallopeptidase 9 (MMP-9) protein was 0.85, 0.72 and $0.57(\mathrm{p}<0.05)$, The relative expression levels of heat shock protein 90 (Hsp90) in drug treated groups $(2 \mu \mathrm{m}, 4 \mu \mathrm{m}, 6$ $\mu \mathrm{m})$ were $0.98,0.95$ and 0.93 respectively and the statistical analysis results showed that there was no significant difference $(\mathrm{p}<0.05)$. Compared with free curcumin liposomes, the cytotoxicity of free curcumin was the lowest, which was consistent with the results of inhibition of cell proliferation by conventional drug loaded nanoparticles, which may be caused by different cell uptake mechanisms. In vitro experiments, free curcumin is directly absorbed by tumor cells through passive diffusion, so as to quickly enter tumor cells and play a therapeutic effect, while long circulating liposomes mainly enter the cells through slow endocytosis and then release the drug from the liposome can play a role. However, rabies virus glycoprotein derived peptide (RDP) targeted long circulating liposomes enter cells rapidly through receptormediated endocytosis and the cell uptake 


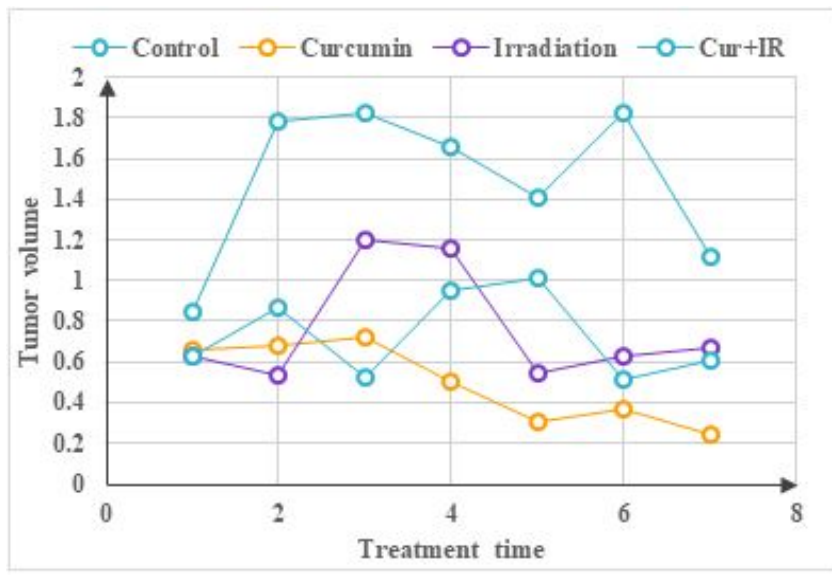

Fig. 4: The effect of different concentrations of curcumin on enzyme activity in glioma cells

is larger than that of non-targeted long circulating liposomes. With the prolongation of in vitro action time, the efficacy of RDP targeted long circulating liposomes is gradually enhanced. As one of the characteristic changes of tumor cells, the metabolic changes of tumor cells are of great significance to the occurrence and development of tumor cells. In some tumor cells, energy metabolism reprogramming occurs due to changes in tumor microenvironment and abnormal expression of cell signaling pathways. In patients with glioma, the glycolysis rate of tumor tissue is three times higher than that of normal brain tissue.

Because ROS can modify the translated protein and cause lipid peroxidation to damage the cell membrane and can directly interact with DNA to cause DNA damage, ROS has long been regarded as a harmful molecule in cells. The ROS produced by the NADPH oxidase family is the second messenger of intracellular signal transduction like reactive nitrogen, which mediates many signal pathways in the cell and is important for cell growth, migration, differentiation and gene expression. Regulatory role, but its specific mechanism of action is not very clear. Human RPE cells are in a high oxidative environment for a long time and are therefore extremely vulnerable to oxidative damage. When the cells endogenous antioxidant system cannot remove excess ROS, excess ROS can upregulate and activate the nuclear transcription factor NF- $\kappa B$ in RPE cells. NF- $\kappa \mathrm{B}$ can increase the expression of TNF- $\alpha$, IL-1 $\beta$ and other cellular inflammatory factors. At the same time, TNF- $\alpha$, IL- $1 \beta$, etc. can also activate NF$\kappa \mathrm{B}$, thereby forming positive feedback and accelerating RPE cell damage. Because diabetic retinopathy (DR) is essentially an inflammatory disease, TNF- $\alpha$ and IL- $1 \beta$, as important inflammatory factors in the human body, play an important role in the development of DR. TNF- $\alpha$ can increase the permeability of retinal capillaries and promote abnormal proliferation of vascular endothelium. At the same time, it can participate in the apoptosis process of RPE cells through the NF- $\mathrm{KB}$ pathway. IL-1 $\beta$ is the IL subtype in which RPE cells play a major role in DR patients. It can cause white blood cell aggregation, promote the inflammatory response of RPE cells and can also affect the normal function of mitochondria in RPE cells and promote RPE cells to undergo apoptosis (fig. 4).

This article mainly studied the related process of curcumin inducing human glioma cell apoptosis by promoting ROS production. The MTT experiment can confirm that curcumin has the effect of inhibiting the proliferation of glioma U87 cells and the Annexin V/ PI double staining experiment verifies the existence of apoptosis in glioma U87 cells treated with curcumin from both quantitative and qualitative aspects. In the end, the western blot method was used to detect apoptosis related proteins, which in turn verified that curcumin can induce apoptosis in human glioma U87 cells. The apoptosis mechanism is that curcumin can induce the expression of $\mathrm{Bcl}-2$-associated $\mathrm{X}$ protein (BAX) and caspase-3, poly ADP ribose polymerase (PARP) genes. The down-regulation of $\mathrm{Bcl}-2$ gene expression and the up-regulation of B-cell lymphoma 2 (Bcl-2) gene expression promoted U87 cell apoptosis. Flow cytometry was used to detect that the tumor cells and they were treated with $10 \mu \mathrm{mol} / \mathrm{l}$, $20 \mu \mathrm{mol} / \mathrm{l}$ curcumin and $100 \mu \mathrm{mol} / 1 \mathrm{H}_{2} \mathrm{O}_{2}$ for $24 \mathrm{~h}$, the mitochondrial membrane potential decreased and the mitochondria were damaged. After $10 \mu \mathrm{mol} / 1,20$ $\mu \mathrm{mol} / 1$ curcumin was treated alone or combined with $100 \mu \mathrm{mol} / 1 \mathrm{H}_{2} \mathrm{O}_{2}$ for $2 \mathrm{~h}$, the overall active oxygen level of cells increased and after $6 \mathrm{~h}$, the overall active oxygen level of cells decreased. It shows that biological antioxidants cause the overall active oxygen level of cells to increase in a short time.

Overexpression of specific drug targets, mutations, drug inactivation and drug outflow from cells may all be the mechanisms of drug resistance. Curcumin can regulate different molecular targets, which has attracted people's attention. Curcumin is non-toxic to regulate various molecular targets, so that curcumin can be realized as an important means of adjuvant radiotherapy and chemotherapy. The standard treatment mode of curcumin, compared with other treatment modes, provides a favorable clinical management mode. The growth of transplanted tumors was significantly inhibited after treatment, suggesting that curcumin 
has a significant direct inhibitory effect on intracranial gliomas. In terms of mechanism research, the extracellular signal regulated kinase (ERK) signaling pathway was also inhibited during the treatment of glioma by intratumoral injection of curcumin, suggesting that it mediates the process of intratumoral injection of curcumin in the treatment of glioma. It can be seen that the ERK signaling pathway is one of the important ways for curcumin to inhibit human glioma in vivo or in vitro.

The increase of curcumin concentration significantly reduced the expression of NF-kB-p65 in RPE cells induced by high glucose. Therefore, curcumin inhibits the overexpression of VEGF and TGF- $\beta 1$ in RPE cells under high glucose culture, which is related to the inhibition of the over activation of NF- $\kappa \mathrm{B}$ in RPE cells. In summary, curcumin can reduce the oxidative stress and inflammation of RPE cells under high glucose conditions by blocking the excessive activation of the $\mathrm{NF}-\kappa \mathrm{B}$ pathway and reduce the expression of fibrosis related factors in RPE cells. Therefore, curcumin can inhibit the oxidative damage and fibrosis of RPE cells induced by high glucose, protect the normal structure and function of RPE cells and provide new ideas for the treatment of DR.

\section{Author's contributions:}

Yu Zhou and Li Liu contributed equally to this work.

\section{Conflict of interests:}

The authors declared no conflict of interest.

\section{REFERENCES}

1. Engwer C, Knappitsch M, Surulescu C. A multiscale model for glioma spread including cell-tissue interactions and proliferation. Math Biosci Eng 2016;13(2):443-60.

2. Yu J, Guo M, Wang T, Li X, Wang D, Wang X, et al. Inhibition of cell proliferation, migration and invasion by a gliomatargeted fusion protein combining the p53 C terminus and MDM2-binding domain. Cell Prolif 2016;49(1):79-89.

3. Zhou Y, Peng Y, Liu M, Jiang Y. MicroRNA-181b inhibits cellular proliferation and invasion of glioma cells via targeting Sal-like protein 4. Oncol Res 2017;25(6):947-7.

4. Kim EH, Yoon MJ, Kim SU, Kwon TK, Sohn S, Choi KS. Arsenic trioxide sensitizes human glioma cells, but not normal astrocytes, to TRAIL-induced apoptosis via CCAAT/ enhancer-binding protein homologous protein-dependent DR5 up-regulation. Cancer Res 2008;68(1):266-75.

5. Buckner JC, Shaw EG, Pugh SL, Chakravarti A, Gilbert MR, Barger GR, et al. Radiation plus procarbazine, CCNU and vincristine in low-grade glioma. N Engl J Med 2016;374(14):1344-55.

6. He C, Jiang B, Ma J, Li Q. Aberrant NEAT 1 expression is associated with clinical outcome in high grade glioma patients. APMIS 2016;124(3):169-74.

7. Zhang R, Pang B, Xin T, Guo H, Xing Y, Xu S, et al. Plasma miR-221/222 family as novel descriptive and prognostic biomarkers for glioma. Mol Neurobiol 2016;53(3):1452-60.

8. Zhu Y, Zhao H, Rao M, Xu S. MicroRNA-365 inhibits proliferation, migration and invasion of glioma by targeting PIK3R3. Oncol Rep 2017;37(4):2185-92.

9. Hu J, Sun T, Wang H, Chen Z, Wang S, Yuan L, et al. MiR215 is induced post-transcriptionally via HIF-Drosha complex and mediates glioma-initiating cell adaptation to hypoxia by targeting KDM1B. Cancer Cell 2016;29(1):49-60.

10. Baumert BG, Hegi ME, van den Bent MJ, von Deimling A, Gorlia T, Hoang-Xuan K, et al. Temozolomide chemotherapy versus radiotherapy in high-risk low-grade glioma (EORTC 22033-26033): a randomised, open-label, phase 3 intergroup study. Lancet Oncol 2016;17(11):1521-32.

11. Hamard L, Ratel D, Selek L, Berger F, van Der Sanden B, Wion D. The brain tissue response to surgical injury and its possible contribution to glioma recurrence. J Neurooncol 2016;128(1):1-8.

12. Lan J, Xue Y, Chen H, Zhao S, Wu Z, Fang J, et al. Hypoxiainduced miR-497 decreases glioma cell sensitivity to TMZ by inhibiting apoptosis. FEBS Lett 2014;588(18):3333-9. 